\title{
Life-Space mobility and clinical outcomes in COPD
}

This article was published in the following Dove Press journal:

International Journal of COPD

\author{
Anand S lyer ${ }^{1-3}$ \\ James MWells 1,3,4 \\ Surya $P$ Bhatt ${ }^{1,3}$ \\ deNay P Kirkpatrick ${ }^{1,3}$ \\ Patricia Sawyer ${ }^{5}$ \\ Cynthia J Brown ${ }^{4,5}$ \\ Richard M Allman 6 \\ Marie A Bakitas ${ }^{5,7,8}$ \\ Mark T Dransfield 1,3,4
}

'Department of Medicine, Division of Pulmonary, Allergy, and Critical

Care Medicine, University of Alabama at Birmingham, Birmingham, AL,

USA; ${ }^{2}$ Health Services, Outcomes, and Effectiveness Research Training Program, University of Alabama at Birmingham, Birmingham, AL, USA;

${ }^{3}$ Department of Medicine, Lung Health Center, University of Alabama at Birmingham, Birmingham, AL, USA; ${ }^{4}$ Veterans Affairs Medical Center, Birmingham, AL, USA; ${ }^{5}$ Department of Medicine, Division of Gerontology, Geriatrics, and Palliative Care, University of Alabama at Birmingham, Birmingham, AL, USA; ${ }^{6}$ Department of Medicine, George Washington University School of Medicine, Washington, DC, USA; ${ }^{7}$ Department of Medicine, Center for Palliative and Supportive Care, Division of Geriatrics, Gerontology, and Palliative Care Medicine, University of Alabama at Birmingham, Birmingham, AL, USA; ${ }^{8}$ School of Nursing, University of Alabama at Birmingham, Birmingham, AL, USA

Correspondence: Anand S lyer Division of Pulmonary, Allergy and Critical Care Medicine, University of Alabama at Birmingham, 1900 University Boulevard THT4I3, Birmingham, AL 35294, USA

Email aiyer@uabmc.edu
Background: Social isolation is a common experience in patients with COPD but is not captured by existing patient-reported outcomes, and its association with clinical outcomes is unknown. Methods: We prospectively enrolled adults with stable COPD who completed the University of Alabama at Birmingham Life Space Assessment (LSA) (range: 0-120, restricted Life-Space mobility: $\leq 60$ and a marker of social isolation in older adults); six-minute walk test (6MWT), and the University of California at San Diego Shortness of Breath Questionnaire, COPD Assessment Test, and Hospital Anxiety and Depression Scale. The occurrence of severe exacerbations (emergency room visit or hospitalization) was recorded by review of the electronic record up to 1 year after enrollment. We determined associations between Life-Space mobility and clinical outcomes using regression analyses.

Results: Fifty subjects had a mean \pm SD \%-predicted $\mathrm{FEV}_{1}$ of $42.9 \pm 15.5$, and $23(46 \%)$ had restricted Life-Space mobility. After adjusting for age, gender, \%-predicted $\mathrm{FEV}_{1}$, comorbidity count, inhaled corticosteroid/long-acting beta 2 -agonist use, and prior cardiopulmonary rehabilitation, subjects with restricted Life-Space had an increased risk for severe exacerbations (adjusted incidence rate ratio $4.65,95 \%$ CI $1.19-18.23, P=0.03)$. LSA scores were associated with 6MWD $(R=0.50, P<0.001)$, dyspnea $(R=-0.58, P<0.001)$, quality of life $(R=-0.34$, $P=0.02)$, and depressive symptoms $(R=-0.39, P=0.005)$.

Conclusion: Restricted Life-Space mobility predicts severe exacerbations and is associated with reduced exercise tolerance, more severe dyspnea, reduced quality of life, and greater depressive symptoms.

Keywords: COPD, patient-reported outcomes, acute exacerbation of COPD

\section{Introduction}

COPD is a progressive disease marked by episodic exacerbations, reduced exercise tolerance, debilitating dyspnea, and poor quality of life. ${ }^{1,2}$ Existing patient-reported outcomes (PROs) capture these well-known aspects of the disease, ${ }^{3}$ but patients with COPD also report social isolation as a major contributor to poor quality of life, and no instrument has been validated to capture this important patient experience in COPD, nor is it routinely considered a therapeutic target. ${ }^{4,5}$

Restricted Life-Space mobility, defined as a score of $\leq 60$ units on the University of Alabama at Birmingham (UAB) Life Space Assessment (LSA), is a marker of social isolation in community-dwelling older adults, with nearly $80 \%$ of UAB Study of Aging participants who had restricted Life-Space mobility also reporting being minimally or not socially active. ${ }^{6}$ Restricted Life-Space mobility predicts mortality, nursing home admission, and reduced quality of life in this population. ${ }^{7-9}$ In an analysis by Garcia et al, ${ }^{10}$ LifeSpace mobility was found to be reduced in adults with COPD compared to those without. However, associations between restricted Life-Space mobility and severe exacerbations of 
COPD have not been prospectively examined, and relationships with other clinically relevant outcomes have not been explored. We hypothesized that restricted Life-Space mobility would predict severe exacerbations of COPD and would be associated with reduced exercise tolerance, more severe dyspnea, worse quality of life, and greater psychological symptoms.

\section{Methods}

\section{Study design and population}

We conducted a prospective cohort study of adults $\geq 40$ years of age with COPD recruited from a convenience sample of patients who attended a weekly, half-day subspecialty pulmonary clinic at UAB in Birmingham, Alabama, USA, between February 2016 and February 2017. We included subjects with a clinical diagnosis of COPD on routine spirometry $\left(\mathrm{FEV}_{1} / \mathrm{FVC}<0.70\right.$ and $\mathrm{FEV}_{1}<80 \%$ predicted) collected within the 2 years preceding enrollment. We excluded subjects with mild COPD ( $\mathrm{FEV}_{1} \geq 80 \%$ ), those who reported an acute exacerbation of COPD requiring steroids or antibiotics in the 30 days prior to enrollment, subjects with other primary lung diseases (eg, asthma, sarcoidosis, interstitial lung disease) or lung cancer, and subjects unable to perform the six-minute walk test owing to musculoskeletal or neurological constraints impeding ambulation. All subjects provided written informed consent to participate, and the Institutional Review Board for the University of Alabama at Birmingham approved the study (IRB-160107005).

\section{Measures and data collection}

We grouped subjects by Global Initiative for Chronic Obstructive Lung Disease (GOLD) spirometric grades as follows: GOLD II $\left(50 \% \leq \mathrm{FEV}_{1}<80 \%\right.$ predicted $)$, GOLD III $\left(30 \% \leq \mathrm{FEV}_{1}<50 \%\right.$ predicted $)$, and GOLD IV $\left(\mathrm{FEV}_{1}<30 \%\right)$. We generated a comorbidity count from the following physician-diagnosed comorbidities in the electronic record: coronary artery disease, diabetes mellitus, congestive heart failure, stroke, osteoarthritis, osteoporosis, hypertension, hyperlipidemia, gastroesophageal reflux disease, stomach ulcers, obesity (body mass index $\geq 30 \mathrm{~kg} / \mathrm{m}^{2}$ ), obstructive sleep apnea, hay fever, and peripheral vascular disease. $^{11}$

Our primary outcome was severe exacerbations of COPD leading to care in the emergency room or requiring hospital admission, assessed by review of the electronic medical record up to 1 year after enrollment. In addition to administering the LSA at baseline, we measured: 1) six-minute walk test (6MWT); 2) University of California at San Diego Shortness of Breath Questionnaire (SOBQ) for dyspnea; ${ }^{12}$
3) COPD Assessment Test (CAT) for quality of life; ${ }^{13}$ and 4) Hospital Anxiety and Depression Scale (HADS) for anxiety and depressive symptoms. ${ }^{14,15}$

\section{UAB LSA}

The LSA is validated for administration in person and by telephone, and scores range from 0 to 120 (Figure 1). In previous analyses, community-dwelling older adults reported being "restricted" with an LSA score $\leq 60 .{ }^{16}$ Purposeful movement through five Life-Space levels, from the room where one sleeps to beyond the town, provides multiple opportunities for social interaction. ${ }^{6}$ For instance, a person with an LSA score of 120 is fully unrestricted and leaves town daily without assistance from another person or assistive device. Someone with an LSA score $\leq 60$ could have a score of zero, indicating never getting out of the bedroom. Alternatively, they could have an LSA score of 60, indicating never getting out of town but driving to town to go shopping twice a week. Moreover, the LSA can detect changes in Life-Space mobility associated with clinically important events such as falls, hospital admissions, and hip fractures, with declines in LSA scores of 3, 10, and nearly 24 points, respectively, expected for these specific events. ${ }^{7,17,18}$ The UAB LSA has no copyright. No permission is required to reproduce the form, and the authors encourage its use in research and clinical settings.

\section{Statistical analysis}

We analyzed descriptive data and reported mean \pm SD for continuous variables and proportions (\%) for categorical variables. We separated the cohort into groups based on restricted $(\leq 60)$ versus unrestricted $(>60)$ Life-Space mobility and compared between-group differences in demographic and clinical characteristics using the chi-squared test, independent $t$-test, or Mann-Whitney $U$-test where appropriate. We examined associations between restricted versus unrestricted Life-Space mobility and the primary outcome of severe exacerbations of COPD using negative binomial regression, adjusting for age, gender, \%-predicted $\mathrm{FEV}_{1}$, comorbidity count, inhaled corticosteroid/long-acting beta $_{2}$-agonist use, and prior cardiopulmonary rehabilitation, which were clinically or statistically significantly associated with severe exacerbations in the bivariate analysis at $P<0.05$. We used linear regression to investigate associations between LSA scores and 6MWT, SOBQ, CAT, and HADS. We used SPSS 23.0 (IBM Corp., Armonk, NY, USA) for statistical analysis. All tests were two sided, and we defined $P<0.05$ as statistically significant. 
The UAB Life Space Assessment

Name:

Date:

\section{These questions refer to your activities just within the past month}

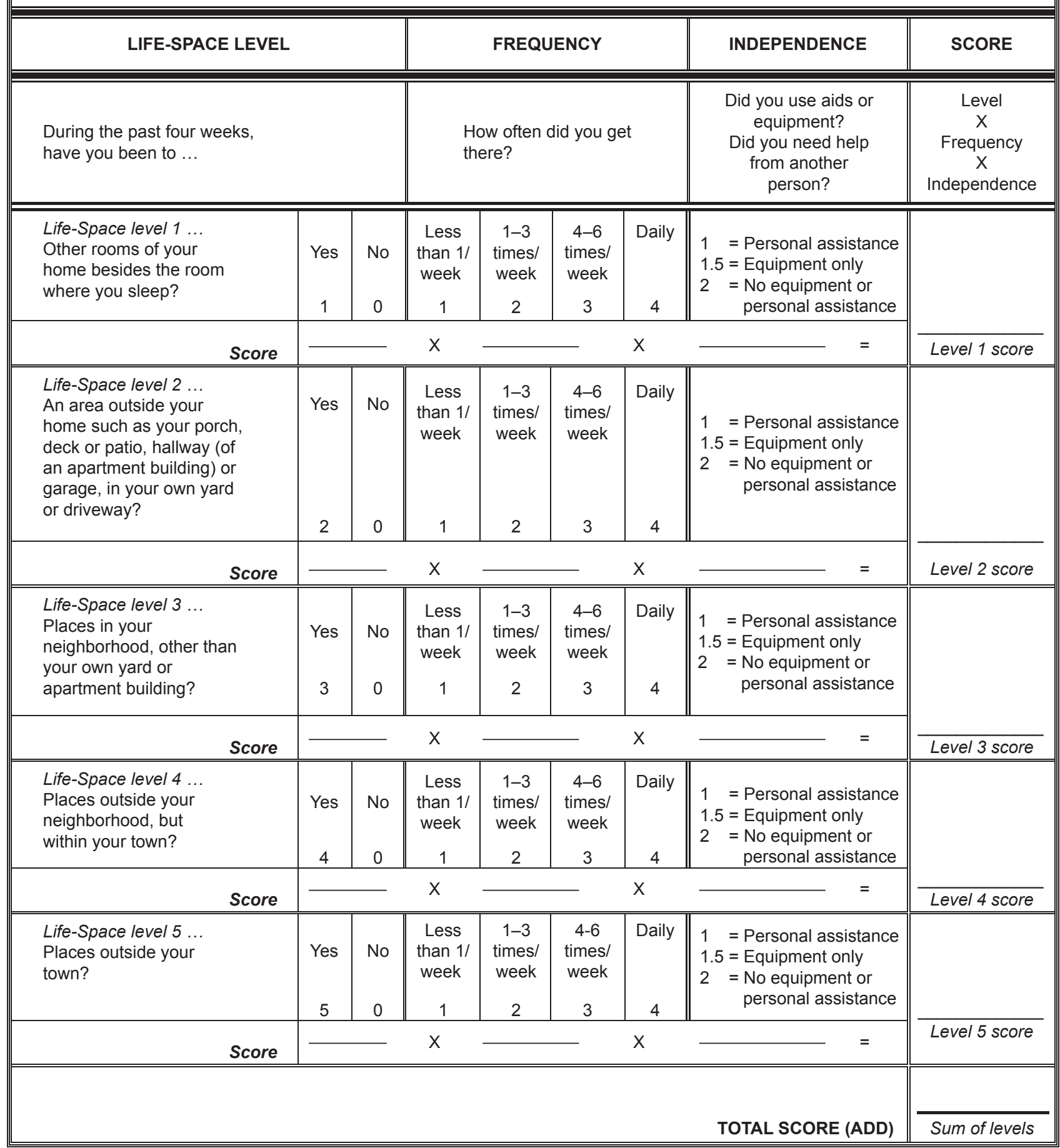

Figure I The UAB LSA.

Notes: The UAB LSA measures Life-Space mobility in the 4 weeks prior to administration through five Life-Space levels from the bedroom where one sleeps to beyond the town. The UAB LSA has no copyright. No permission is required to reproduce the form, and the authors encourage its use in research and clinical settings. Values for distance, frequency, and independence of movement are multiplied to generate a score for each Life-Space level, and these scores are summed to generate a total LSA score. Mobility through a higher Life-Space level automatically means someone moved through lower Life-Space levels, ie, if someone moves through the town, he also travels through the house and the neighborhood. In such a situation, the scores for the lower Life-Space level must be corrected to match those of the immediately higher Life-Space level.

Abbreviations: UAB, University of Alabama at Birmingham; LSA, Life Space Assessment. 


\section{Results}

\section{Study cohort}

We enrolled 50 current and former smokers with stable COPD. Subjects had a mean \pm SD age of $66.2 \pm 9.6$ years and a mean \%-predicted $\mathrm{FEV}_{1}$ of $42.9 \% \pm 15.5 \%$, with $66 \%$ having GOLD III-IV stage COPD (Table 1). Overall, 18 subjects (36\%) were African-American, 23 (46\%) had previously completed cardiopulmonary rehabilitation, 39 (78\%) were taking a long-acting muscarinic antagonist, and 37 (74\%) were taking a inhaled corticosteroid/long-acting beta ${ }_{2}$-agonist.

Subjects had a mean LSA score of 69.9 \pm 28.2 (range 18-120), with 23 (46\%) having restricted Life-Space mobility (LSA score $\leq 60$ ). Compared with subjects who had unrestricted Life-Space mobility, those with restricted Life-Space mobility had more severe airflow limitation $\left(\mathrm{FEV}_{1}\right.$ $\%$-predicted $37.7 \% \pm 11.0 \%$ vs $47.4 \% \pm 17.5 \%, P=0.02$ ). Subjects with restricted Life-Space mobility also had a higher comorbidity count ( $3.1 \pm 1.7$ vs $2.1 \pm 1.5, P=0.03$ ) (Table 1 ).

\section{Life-Space mobility and clinical outcomes}

We recorded 13 severe exacerbations in the year following enrollment. Restricted Life-Space mobility predicted severe exacerbations (unadjusted incidence rate ratio [IRR] 4.99, $95 \% \mathrm{CI} 1.47-16.95, P=0.01$ ), and this association remained significant after adjustment for age, gender, \%-predicted $\mathrm{FEV}_{1}$, comorbidity count, inhaled corticosteroid/long-acting beta $_{2}$-agonist use, and prior cardiopulmonary rehabilitation (adjusted IRR 4.65, 95\% CI 1.19-18.23, $P=0.03$ ). Compared to subjects with unrestricted Life-Space mobility, those with restricted Life-Space mobility had significantly reduced 6MWD (237.2 $\pm 101.8 \mathrm{~m}$ vs $318.8 \pm 68.4 \mathrm{~m}, P=0.002)$, more severe dyspnea by the SOBQ $(70.7 \pm 23.5$ vs $41.9 \pm 25.9$, $P<0.001)$, and worse quality of life by the CAT $(21.5 \pm 7.8$ vs $15.4 \pm 5.8, P=0.003$ ) (Table 1 ). LSA scores were associated with distance on the 6MWT $(R=0.50, P<0.001)$, SOBQ $(-0.58, P<0.001)$, and CAT total score $(R=-0.34$, $P=0.02$ ) (Figure 2).

Table I Characteristics of subjects by restricted Life-Space mobility

\begin{tabular}{|c|c|c|c|c|}
\hline \multirow[t]{2}{*}{ Characteristic } & \multirow{2}{*}{$\begin{array}{l}\text { Overall } \\
(n=50)\end{array}$} & \multicolumn{2}{|c|}{ Restricted Life-Space mobility ${ }^{a}$} & \multirow[t]{2}{*}{$P$-value } \\
\hline & & $\begin{array}{l}\text { No } \\
(n=27)\end{array}$ & $\begin{array}{l}\text { Yes } \\
(n=23)\end{array}$ & \\
\hline Age (years) & $66.2 \pm 9.6$ & $67.7 \pm 9.2$ & $64.3 \pm 9.8$ & 0.21 \\
\hline Ethnicity (African American) & $18(36)$ & $8(30)$ & $10(44)$ & 0.31 \\
\hline Gender (female) & $28(56)$ & $13(48)$ & $15(65)$ & 0.23 \\
\hline Current smoking & $14(28)$ & $7(26)$ & $7(30)$ & 0.72 \\
\hline Comorbidity count ${ }^{\mathrm{b}}$ & $2.6 \pm 1.7$ & $2.1 \pm 1.5$ & $3.1 \pm 1.7$ & 0.03 \\
\hline Supplemental oxygen & $15(30)$ & $7(26)$ & $8(35)$ & 0.50 \\
\hline$\geq \mid$ severe exacerbation in year prior ${ }^{c}$ & $14(28)$ & $5(19)$ & $9(39)$ & 0.11 \\
\hline $\mathrm{FEV}_{1}$ (\%-predicted) & $42.9 \pm 15.5$ & $47.4 \pm 17.5$ & $37.7 \pm 11.0$ & 0.02 \\
\hline GOLD stage & & & & 0.24 \\
\hline GOLD II & $17(34)$ & $12(36.4)$ & $5(29.4)$ & \\
\hline GOLD III & $20(40)$ & $9(33)$ & II (48) & \\
\hline GOLD IV & $13(26)$ & $6(22)$ & $7(30)$ & \\
\hline Prior cardiopulmonary rehabilitation & $23(46)$ & $9(33)$ & $14(61)$ & 0.05 \\
\hline \multicolumn{5}{|l|}{ Inhaler use } \\
\hline Short-acting beta-agonist & $46(92)$ & $24(89)$ & $22(96)$ & 0.38 \\
\hline Long-acting muscarinic antagonist & $39(78)$ & $21(78)$ & $18(78)$ & 0.97 \\
\hline Inhaled corticosteroid/long-acting beta ${ }_{2}$-agonist & $37(74)$ & $17(63)$ & $20(87)$ & 0.05 \\
\hline \multicolumn{5}{|l|}{ Outcomes } \\
\hline Distance on the 6MWT $(\mathrm{m})$ & $281.2 \pm 94.0$ & $318.8 \pm 68.4$ & $237.2 \pm 101.8$ & 0.002 \\
\hline SOBQ & $55.1 \pm 28.6$ & $41.9 \pm 25.9$ & $70.7 \pm 23.5$ & $<0.001$ \\
\hline CAT total score & $18.2 \pm 7.4$ & $15.4 \pm 5.8$ & $21.5 \pm 7.8$ & 0.003 \\
\hline \multicolumn{5}{|l|}{ HADS } \\
\hline HADS-Depression & $5.6 \pm 4.3$ & $4.0 \pm 3.8$ & $7.6 \pm 4.1$ & 0.002 \\
\hline Elevated depressive symptoms ${ }^{d}$ & $15(30)$ & $4(15)$ & II (48) & 0.01 \\
\hline HADS-Anxiety & $6.2 \pm 4.3$ & $5.1 \pm 4.1$ & $7.4 \pm 4.3$ & 0.06 \\
\hline Elevated anxiety symptoms ${ }^{d}$ & $19(38)$ & $7(26)$ & $12(52)$ & 0.06 \\
\hline
\end{tabular}

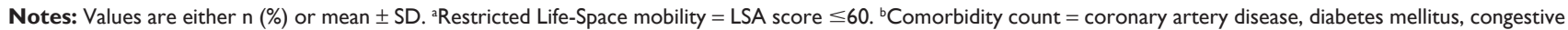
heart failure, stroke, osteoarthritis, osteoporosis, hypertension, hyperlipidemia, gastroesophageal reflux disease, stomach ulcers, obesity (body mass index $\geq 30 \mathrm{~kg} / \mathrm{m}^{2}$ ),

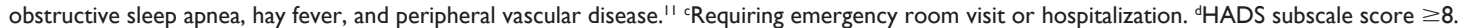

Abbreviations: FEV , forced expiratory volume in I second; GOLD, Global Initiative for Chronic Obstructive Lung Disease; 6MWT, six-minute walk test; SOBQ, University of California at San Diego Shortness of Breath Questionnaire; CAT, COPD Assessment Test; HADS, Hospital Anxiety and Depression Scale; LSA, Life Space Assessment. 


\section{Life-Space mobility and outcomes}
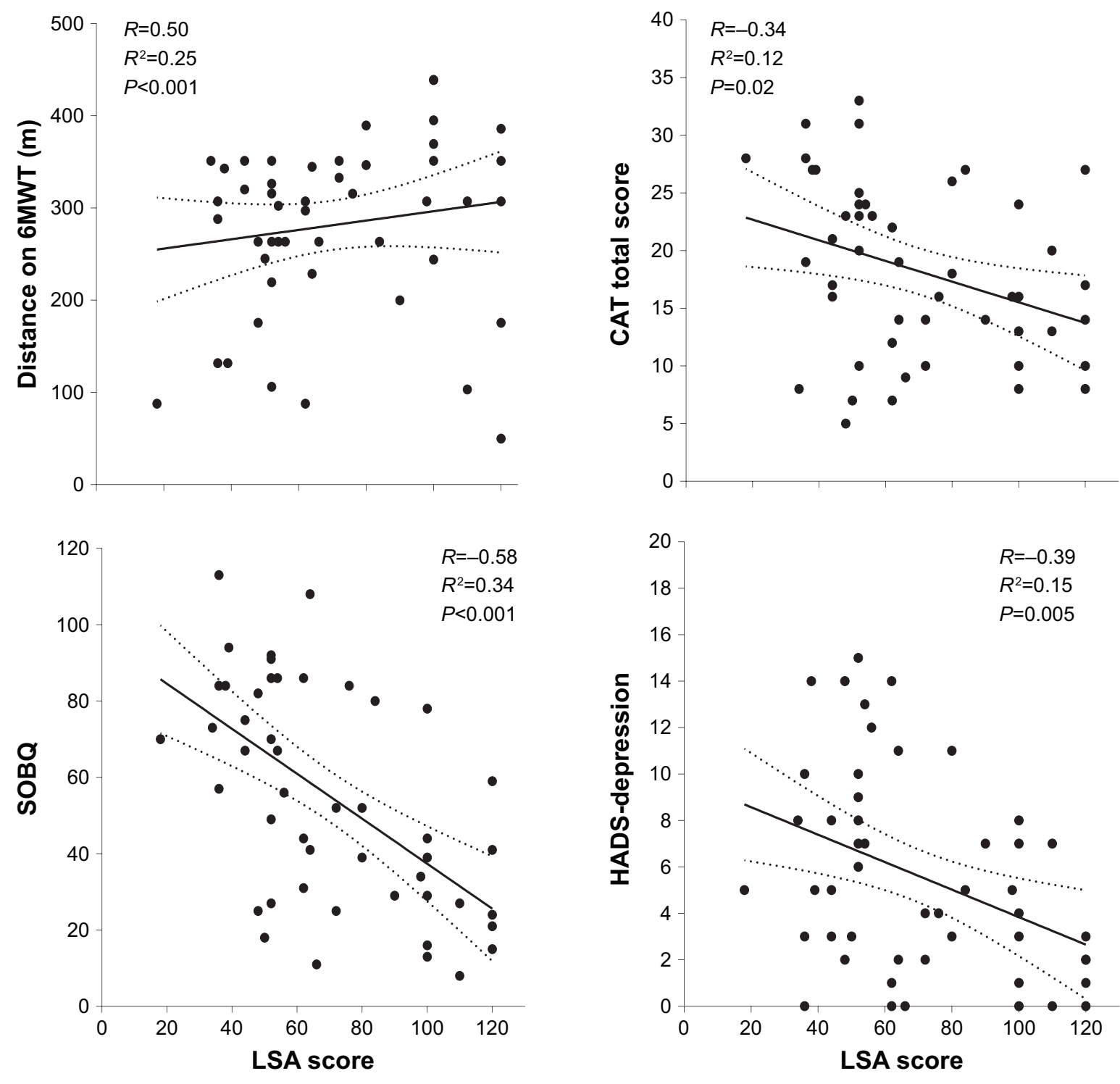

$\leftarrow$ Restricted Unrestricted $\rightarrow$

$\leftarrow$ Restricted Unrestricted $\rightarrow$

Figure 2 Life-Space mobility and clinical outcomes in COPD.

Note: Linear regression analyses between continuous LSA score and distance on 6MWT, SOBQ, CAT, and HADS-Depression.

Abbreviations: LSA, Life Space Assessment; 6MWT, Six-minute walk test; SOBQ, University of California at San Diego Shortness of Breath Questionnaire; CAT, COPD Assessment Test; HADS, Hospital Anxiety and Depression Scale.

Thirty-eight percent of the cohort had clinically elevated anxiety symptoms by HADS-Anxiety $\geq 8$, and $30 \%$ had clinically elevated depressive symptoms by HADSDepression $\geq 8$ (Table 1). ${ }^{14}$ Subjects with restricted LifeSpace mobility had three times the frequency of elevated depressive symptoms of those with unrestricted Life-Space mobility ( $48 \%$ vs $15 \%, P=0.01$ ) (Table 1 ), and LSA scores were inversely associated with HADS-Depression $(R=-0.39$, $P=0.02$ ) (Figure 2 ). We found no statistically significant association between LSA score and HADS-Anxiety score $(P=0.11)$.

\section{Discussion}

Nearly half of subjects from a sample of adults with stable COPD have restricted Life-Space mobility, a marker of social isolation and predictor of poor outcomes in community-dwelling older adults. Restricted Life-Space mobility independently predicts severe exacerbations of COPD and 
is associated with multiple clinically relevant outcomes, including reduced exercise tolerance, more severe dyspnea, worse quality of life, and depressive symptoms. These results suggest that LSA may be a clinically relevant PRO to aid in risk stratification in COPD.

Only two studies have explored Life-Space mobility in chronic lung disease. First, Gottlieb et al investigated Life-Space mobility in cystic fibrosis and found that airflow limitation and all-cause hospitalizations in the year prior to enrollment were associated with restricted Life-Space mobility. ${ }^{19}$ However, that study did not prospectively examine the impact of restricted Life-Space mobility on respiratory exacerbations. ${ }^{19}$ Second, Garcia et al examined Life-Space mobility in a cohort of older adults with and without COPD and found that Life-Space mobility was more restricted in COPD patients compared to individuals without COPD. ${ }^{10}$ However, that study did not enroll patients with severe airflow limitation and did not explore the impact of restricted Life-Space mobility on exacerbations, quality of life, and psychological symptoms. ${ }^{10}$ Our analysis is the first exploration of the LSA in a well-defined cohort of subjects with moderate to very severe COPD, and our data suggest that the LSA could be an important new PRO with applications in both clinical and research settings. The LSA could be used to target patients at high risk of exacerbation for enrollment into clinical trials, to select patients who may have reduced exercise tolerance and thus need measurement of 6MWT, and to guide referral to palliative care based on a high symptom burden.

The LSA has been validated in community-dwelling older adults, and multiple publications support its clinical validity in that population. ${ }^{16}$ In addition to its association with poor outcomes in older adults, such as mortality, ${ }^{7}$ hospitalization and emergency room visits, ${ }^{20}$ and nursing home admission, ${ }^{8}$ restricted Life-Space mobility is associated with frailty, unintentional weight loss, cognitive decline, and falls, and precedes limitations in activities of daily living in this population. ${ }^{18,21-25}$ Many COPD patients also experience these common features of aging and frequently limit participation in social activities; however, this is rarely assessed. ${ }^{4}$ In fact, the frequency of restricted Life-Space mobility in our cohort (46\%) exceeded that of community-dwelling older adults in the UAB Study of Aging (41\%) despite our cohort being approximately 10 years younger on average. ${ }^{16}$ In addition, we found that subjects with stable COPD and restricted Life-Space mobility had significantly worse quality of life and greater depressive symptoms, results that echo findings from other international studies which have found significant associations between Life-Space mobility and domains of well-being in older adults. ${ }^{26,27}$ Though we did not find a statistically significant bivariate association between anxiety symptoms and Life-Space mobility, anxiety symptoms were twice as frequent in subjects with restricted Life-Space mobility than in those without, suggesting that social mobility plays an important role in emotional well-being.

Restricted Life-Space mobility predicts severe exacerbations in COPD even after adjustment for age, gender, airflow limitation, comorbidities, inhaler use, and cardiopulmonary rehabilitation, suggesting that measurement of Life-Space mobility provides independent prognostic information. PROs aimed at measuring the comprehensive impact of COPD and predicting health care utilization are lengthy and time consuming. ${ }^{28}$ Furthermore, PRO development and validation often take years, and existing PROs ultimately offer limited additional predictive benefit over existing risk scores such as the BODE (Body Mass Index, airflow Obstruction, Dyspnea, and Exercise) index. ${ }^{5,29}$ In contrast, the LSA has a number of advantages including the fact it is brief, easy to use, and not effort dependent. Though the mechanism linking restricted Life-Space mobility to severe exacerbations remains to be elucidated, it is clearly a fundamental aspect of the patient experience not captured by current COPD-specific PROs. ${ }^{6}$

Our study is limited by its single-center design and small sample size, which may affect its generalizability. Furthermore, we only had access to utilization data from our institution and did not have access to moderate exacerbations. However, our hospital is a large tertiary referral center with a comprehensive COPD management program, ${ }^{30}$ and it is unlikely that patients who visited our subspecialty clinic were admitted to other hospitals in the area.

In conclusion, restricted Life-Space mobility is a powerful predictor of severe exacerbations in COPD and is associated with greater symptom burden. The LSA could be routinely measured in clinical and research settings to guide COPD risk stratification and to discover potential unmet needs. Further validation of the LSA in larger cohorts is warranted to determine the associations between restricted Life-Space mobility and moderate exacerbations.

\section{Author contributions}

All authors contributed toward data analysis, drafting and revising the paper and agree to be accountable for all aspects of the work.

\section{Disclosure}

ASI reports support from UAB Patient Centered Outcomes Research [K12HS023009]. SPB acknowledges support from 
the NIH [K23HL133438]. JMW acknowledges support from the NIH [K08HL123940]. RMA has no conflicts of interest to report. MAB is supported by NINR 1 R01 [NR01366501A1]. MTD reports grants from the NIH and Department of Defense, consulting fees from AstraZeneca, Genentech, GlaxoSmithKline, and PneumRx/BTG, and contracted clinical trials from AstraZeneca, Boehringer Ingelheim, GlaxoSmithKline, Novartis, PneumRx/BTG, Pulmonx, and Yungjin. This study was supported in part by a grant from the NIA [R01 AG015062] to CJB. dPK and PS have no conflicts to disclose.

\section{References}

1. Han MK, Martinez CH, Au DH, et al. Meeting the challenge of COPD care delivery in the USA: a multiprovider perspective. Lancet Respir Med. 2016;4(6):473-526.

2. Oga T, Nishimura K, Tsukino M, Sato S, Hajiro T, Mishima M. Longitudinal deteriorations in patient reported outcomes in patients with COPD. Respir Med. 2007;101(1):146-153.

3. Vogelmeier CF, Criner GJ, Martinez FJ, et al. Global Strategy for the Diagnosis, Management, and Prevention of Chronic Obstructive Lung Disease 2017 Report. GOLD Executive Summary. Am J Respir Crit Care Med. 2017;195(5):557-582.

4. Schroedl CJ, Yount SE, Szmuilowicz E, Hutchison PJ, Rosenberg SR, Kalhan R. A qualitative study of unmet healthcare needs in chronic obstructive pulmonary disease. A potential role for specialist palliative care? Ann Am Thorac Soc. 2014;11(9):1433-1438.

5. Strassmann A, Frei A, Haile SR, Ter Riet G, Puhan MA. Commonly used patient-reported outcomes do not improve prediction of COPD exacerbations: a multicenter $4 \frac{1}{2}$ year prospective cohort study. Chest. 2017;152(6):1179-1187.

6. Sawyer P, Allman RM. Resilience in mobility in the context of chronic disease and aging. In: Fry PS, Keyes CLM, editors. New Frontiers in Resilient Aging: Life-Strengths and Well-Being in Late Life. Cambridge: Cambridge University Press; 2010:310-339.

7. Kennedy RE, Sawyer P, Williams CP, et al. Life-Space Mobility Change Predicts 6-Month Mortality. J Am Geriatr Soc. 2017;65(4):833-838.

8. Sheppard KD, Sawyer P, Ritchie CS, Allman RM, Brown CJ. Lifespace mobility predicts nursing home admission over 6 years. $J$ Aging Health. 2013;25(6):907-920.

9. Rantakokko M, Portegijs E, Viljanen A, Iwarsson S, Kauppinen M, Rantanen T. Changes in life-space mobility and quality of life among community-dwelling older people: a 2-year follow-up study. Qual Life Res. 2016;25(5):1189-1197.

10. Garcia IFF, Tiuganji CT, Simões M, Santoro IL, Lunardi AC. Systemic effects of chronic obstructive pulmonary disease in young-old adults' life-space mobility. Int J Chron Obstruct Pulmon Dis. 2017; 12:2777-2785.

11. Putcha N, Puhan MA, Drummond MB, et al. A simplified score to quantify comorbidity in COPD. PLoS One. 2014;9(12):e114438.

12. Ries AL. Minimally clinically important difference for the UCSD Shortness of Breath Questionnaire, Borg Scale, and Visual Analog Scale. COPD. 2005;2(1):105-110.

13. Kon SS, Canavan JL, Jones SE, et al. Minimum clinically important difference for the COPD Assessment Test: a prospective analysis. Lancet Respir Med. 2014;2(3):195-203.
14. Zigmond AS, Snaith RP. The hospital anxiety and depression scale. Acta Psychiatr Scand. 1983;67(6):361-370.

15. Puhan MA, Frey M, Büchi S, Schünemann HJ. The minimal important difference of the hospital anxiety and depression scale in patients with chronic obstructive pulmonary disease. Health Qual Life Outcomes. 2008;6:46.

16. Allman RM, Sawyer P, Roseman JM. The UAB Study of Aging: background and insights into life-space mobility among older Americans in rural and urban settings. Aging Health. 2006;2(3):417-429.

17. Brown CJ, Roth DL, Allman RM, Sawyer P, Ritchie CS, Roseman JM Trajectories of life-space mobility after hospitalization. Ann Intern Med. 2009;150(6):372-378.

18. Lo AX, Brown CJ, Sawyer P, Kennedy RE, Allman RM. Life-space mobility declines associated with incident falls and fractures. $J \mathrm{Am}$ Geriatr Soc. 2014;62(5):919-923.

19. Gottlieb ER, Smith EC, Wolfenden LL, Allman RM, Tangpricha V. Life-space mobility is associated with frequency of hospitalization in adults with cystic fibrosis. Clin Respir J. 2011;5(4):245-251.

20. Lo AX, Flood KL, Kennedy RE, et al. The association between lifespace and health care utilization in older adults with heart failure J Gerontol A Biol Sci Med Sci. 2015;70(11):1442-1447.

21. Portegijs E, Rantakokko M, Viljanen A, Sipilä S, Rantanen T. Is frailty associated with life-space mobility and perceived autonomy in participation outdoors? A longitudinal study. Age Ageing. 2016;45(4): 550-553.

22. Ritchie CS, Locher JL, Roth DL, Mcvie T, Sawyer P, Allman R. Unintentional weight loss predicts decline in activities of daily living function and life-space mobility over 4 years among community-dwelling older adults. J Gerontol A Biol Sci Med Sci. 2008;63(1):67-75.

23. Poranen-Clark T, von Bonsdorff MB, Rantakokko M, et al. Executive function and life-space mobility in old age. Aging Clin Exp Res. 2018;30(2):145-151.

24. Bentley JP, Brown CJ, Mcgwin G, Sawyer P, Allman RM, Roth DL. Functional status, life-space mobility, and quality of life: a longitudinal mediation analysis. Qual Life Res. 2013;22(7):1621-1632.

25. Portegijs E, Rantakokko M, Viljanen A, Sipilä S, Rantanen T. Identification of older people at risk of ADL disability using the life-space assessment: a longitudinal cohort study. J Am Med Dir Assoc. 2016; 17(5):410-414.

26. Polku H, Mikkola TM, Portegijs E, et al. Life-space mobility and dimensions of depressive symptoms among community-dwelling older adults. Aging Ment Health. 2015;19(9):781-789.

27. Rantakokko M, Portegijs E, Viljanen A, Iwarsson S, Rantanen T. Lifespace mobility and quality of life in community-dwelling older people J Am Geriatr Soc. 2013;61(10):1830-1832.

28. Puhan MA, Guyatt GH, Goldstein R, et al. Relative responsiveness of the Chronic Respiratory Questionnaire, St. Georges Respiratory Questionnaire and four other health-related quality of life instruments for patients with chronic lung disease. Respir Med. 2007;101(2):308-316.

29. Jones P, Miravitlles M, van der Molen T, Kulich K. Beyond FEV ${ }_{1}$ in COPD: a review of patient-reported outcomes and their measurement. Int J Chron Obstruct Pulmon Dis. 2012;7:697-709.

30. Bhatt SP, Wells JM, Iyer AS, et al. Results of a medicare bundled payments for care improvement initiative for chronic obstructive pulmonary disease readmissions. Ann Am Thorac Soc. 2017;14(5): 643-648. 


\section{Publish your work in this journal}

The International Journal of COPD is an international, peer-reviewed journal of therapeutics and pharmacology focusing on concise rapid reporting of clinical studies and reviews in COPD. Special focus is given to the pathophysiological processes underlying the disease, intervention programs, patient focused education, and self management protocols.

This journal is indexed on PubMed Central, MedLine and CAS. The manuscript management system is completely online and includes a very quick and fair peer-review system, which is all easy to use. Visit http://www.dovepress.com/testimonials.php to read real quotes from published authors.

Submit your manuscript here: http://www.dovepress.com/international-journal-of-chronic-obstructive-pulmonary-disease-journal 